\title{
PSMA Expression Assessed by PET Imaging Is a Required Biomarker for Selecting Patients for Any PSMA-Targeted Therapy
}

\author{
Jeremie Calais and Johannes Czernin
}

David Geffen School of Medicine at UCLA, Los Angeles, California

A mal growth factor receptor 2 (Erb-B2 receptor tyrosine kinase 2 [ERBB2], formerly HER2), and 70\% express estrogen or progesterone receptors. All excised breast cancer tissue is examined for the presence of these molecular markers, as their expression pattern determines the most precise or personalized treatment. For obvious reasons, and despite their limitations, biopsy and surgical tissue sampling to determine target expression have become the undisputed standard of care in breast and many other cancers.

PET imaging has theoretic advantages over biopsies as it can noninvasively survey the entire body for target expression. Imaging probes to measure estrogen, progesterone, androgen, and ERBB2 expression are slowly finding recognition in oncologic practice, as they have the potential to overcome the limitations of invasive tissue sampling.

In prostate cancer, whole-body PET determinations of prostatespecific membrane antigen (PSMA) expression are now established, approved, and available for disease staging and restaging. In addition, limited data suggest that PSMA PET can serve as a predictive biomarker for response to PSMA-targeted therapies.

The VISION trial showed that PSMA-targeted molecular radiotherapy (MRT) can prolong survival and improve quality of life of patients with advanced metastatic castration-resistant prostate cancer (1). This trial selected patients by using PSMA PET as a biomarker. The PSMA PET screen failure rate was $12.6 \%$ $(126 / 1,003)(1)$. Given the high prevalence of PSMA expression in advanced prostate cancer and the PSMA PET screening positivity rate of $87 \%$ in the VISION trial, some have argued that the trial could have been positive even in an unselected population (2).

Other arguments against pretreatment assessments of target expression with PET include the burden of an additional procedure, long waiting times due to limited availability, and high costs of PSMA PET imaging. Here we briefly address each of these arguments:

\section{PSMA Is Near Uniformly Expressed in Prostate Cancer Lesions}

The prevalence of PSMA expression is more than $90 \%$ in prostate cancer (3-6). However, the degree and homogeneity of expression, both determinants of treatment response, vary among and within patients $(7,8)$. Absent or low target expression limits the response to

COPYRIGHT (C) 2021 by the Society of Nuclear Medicine and Molecular Imaging. DOI: 10.2967/jnumed.121.263159
PSMA-targeted therapies $(9,10)$. Studies reported that the whole-body PSMA PET SUV $\mathrm{S}_{\text {mean }}$ correlated with outcomes of patients treated with ${ }^{177}$ Lu-PSMA $(7,11,12)$. In a study conducted at Weill Cornell, fewer than $10 \%$ of patients with no or low PSMA expression at baseline had a significant PSA response to PSMA-targeted MRT (13).

Lesions with low PSMA expression and high glycolytic activity (PSMAnegative $/{ }^{18} \mathrm{~F}-\mathrm{FDG}$-positive) can occur in up to $30 \%$ of metastatic castrationresistant prostate cancer patients referred for PSMA-targeted MRT $(14,15)$. Patients with such aggressive nonresponding lesions have a shorter overall survival in response to PSMA-targeted MRT than those with PSMA-positive $/{ }^{18}$ F-FDG-positive or PSMA-positi$\mathrm{ve} /{ }^{18} \mathrm{~F}-\mathrm{FDG}-$-negative lesions $(15,16)$. At the Peter MacCallum Cancer Centre, Australia, patients are routinely selected for PSMA-targeted MRT by PSMA and ${ }^{18}$ F-FDG PET. The TheraP trial (7) excluded $10 \%$ and $20 \%$ of patients

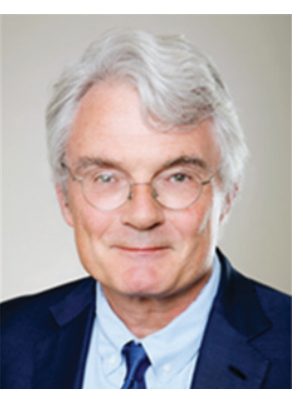

Johannes Czernin

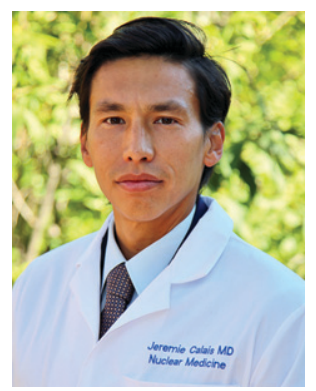

Jeremie Calais based on low lesion PSMA expression or high ${ }^{18} \mathrm{~F}-\mathrm{FDG}$ to PSMA uptake ratios, respectively (14). This careful patient selection likely accounts for the superior progression-free survival of their cohorts in response to MRT when compared with reports from other groups.

In simple terms: no responsible physician would target PSMA if its expression were absent or low in all or some lesions. The non-PSMA-expressing lesions will not respond to PSMA-targeted MRT and will drive patient prognosis. On the basis of these data and our own experience, not characterizing target expression before PSMA-targeted treatment appears as nonsensical as omitting breast cancer receptor phenotyping from the standard work-up of breast cancer patients. Figure 1 depicts a patient with low-PSMAexpressing disease and a poor subsequent treatment response.

\section{Accessibility of PSMA PET Imaging Is Limited}

Limited access to PSMA PET imaging in the United States will be history within the next few months. The U.S. Food and Drug administration has granted a new drug application for 2 compounds 


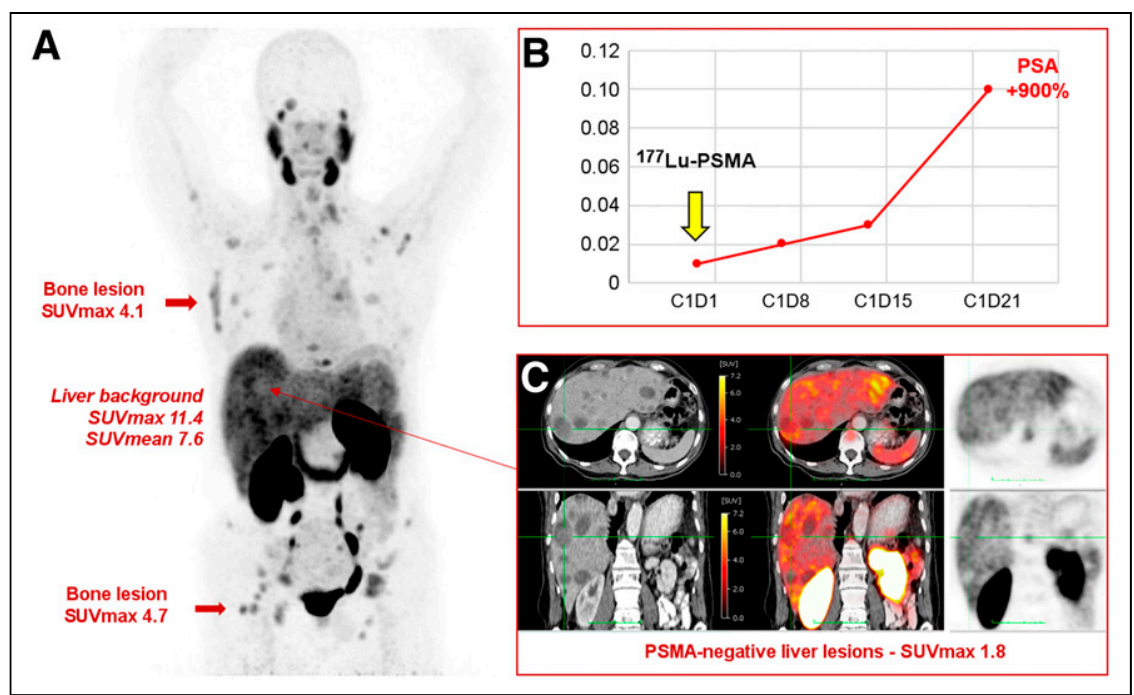

FIGURE 1. PSMA PET/CT of patient with metastatic castration-resistant prostate cancer before treatment with ${ }^{177}$ Lu-PSMA therapy. (A) Maximum-intensity-projection image shows extensive metastatic disease (lymph nodes, bone, liver) with low or no PSMA expression (below, equal to, or just above liver background uptake). (B) Patient received 1 cycle of ${ }^{177}$ Lu-PSMA therapy, with no response and rapid PSA progression. (C) Presence of multiple liver metastases on CT (left panel) without visible PSMA uptake on corresponding fused PET/CT (middle panel) and PET images (right panel). on PSMA imaging await further definition and refinement. However, if we are serious about precision medicine, PSMA imaging must be included in the selection of patients for PSMA-targeted therapies.

\section{DISCLOSURE}

Jeremie Calais reports prior consulting activities outside the submitted work for Advanced Accelerator Applications, Blue Earth Diagnostics, Curium Pharma, GE Healthcare, Janssen, IBA Radiopharma, POINT Biopharma, Progenics, Radiomedix, and Telix Pharmaceuticals. Johannes Czernin is a founder and holds equity in Sofie Biosciences and Trethera Therapeutics and was a consultant for Endocyte Inc. (VISION trial steering committee), Actinium Pharmaceuticals, and Point Biopharma outside the submitted work. Intellectual property is patented by the University of California and licensed to Sofie Biosciences and Trethera Therapeutics. No other potential conflict of interest relevant to this article was reported.
( ${ }^{68}$ Ga-PSMA-11 and ${ }^{18}$ F-PYL) in 2020-2021. Because radiopharmacies will deliver ${ }^{18} \mathrm{~F}$ compounds and PSMA-11 cold kits have become available for local generator-based ${ }^{68} \mathrm{Ga}$ production, PSMA PET will become as universally available as ${ }^{18}$ F-FDG PET.

\section{The Costs of PET/CT Imaging Are High}

Let us assume that 40,000 patients with prostate cancer will undergo ${ }^{177} \mathrm{Lu}$-PSMA treatments per year (17). Let us also assume a high average imaging study cost of $\$ 5,000$ per patient. Imaging costs would then amount to $\$ 200$ million per year. Let us also assume that 1 treatment cycle of ${ }^{177}$ Lu-PSMA will cost $\$ 50,000$ in the United States and that an average of 3 cycles (accounting for treatment discontinuation in nonresponding patients) will be given to each of the 40,000 patients, for a total of 120,000 cycles. Treatment cost would then amount to $\$ 6$ billion per year. Let us finally assume conservatively that 1 of 5 patients $(20 \%)$ would be screened out by PSMA PET/CT imaging because of no, low, or heterogeneous target expression. This screening would reduce the overall treatment costs by $\$ 1.2$ billion per year. Thus, rather than adding expenses, appropriate imaging reduces health-care expenditures for PSMA-targeted theranostics significantly and dramatically, an effect that may not be in the interest of some industry sections but of great benefit for patients and society.

Patients and physicians demand and deserve personalized precision medicine. Radiotheranostics offer the unprecedented opportunity of whole-body image-guided individualized disease management. Imagine how chemotherapy outcomes would improve if whole-body drug biodistribution and target expression could be determined in advance of administration. And imagine how patient selection would improve if treatment failure could be predicted by applying a whole-body imaging biomarker.

We agree with Srinivas and Iagaru (18) that we finally have a predictive whole-body imaging biomarker for response to PSMA-targeted therapies in prostate cancer. Inclusion versus exclusion criteria based

\section{REFERENCES}

1. Sartor O, de Bono J, Chi KN, et al. Lutetium-177-PSMA-617 for metastatic castration-resistant prostate cancer. $N$ Engl J Med. 2021;385:1091-1103.

2. VISION: implementation of lutetium-177-PSMA-617 in metastatic castrationresistant prostate cancer approaches reality. ASCO Daily News website. https:// dailynews.ascopubs.org/do/10.1200/ADN.21.200630/full. Published June 9, 2021. Accessed September 20, 2021

3. Silver DA, Pellicer I, Fair WR, Heston WD, Cordon-Cardo C. Prostate-specific membrane antigen expression in normal and malignant human tissues. Clin Cancer Res. 1997;3:81-85.

4. Sweat SD, Pacelli A, Murphy GP, Bostwick DG. Prostate-specific membrane antigen expression is greatest in prostate adenocarcinoma and lymph node metastases. Urology. 1998;52:637-640.

5. Rüschoff JH, Ferraro DA, Muehlematter UJ, et al. What's behind ${ }^{68}$ Ga-PSMA-11 uptake in primary prostate cancer PET? Investigation of histopathological parameters and immunohistochemical PSMA expression patterns. Eur J Nucl Med Mol Imaging. August 13, 2021 [Epub ahead of print].

6. Bostwick DG, Pacelli A, Blute M, Roche P, Murphy GP. Prostate specific membrane antigen expression in prostatic intraepithelial neoplasia and adenocarcinoma: a study of 184 cases. Cancer. 1998;82:2256-2261.

7. Seifert R, Seitzer K, Herrmann K, et al. Analysis of PSMA expression and outcome in patients with advanced prostate cancer receiving ${ }^{177}$ Lu-PSMA-617 radioligand therapy. Theranostics. 2020;10:7812-7820.

8. Mannweiler S, Amersdorfer P, Trajanoski S, Terrett JA, King D, Mehes G. Heterogeneity of prostate-specific membrane antigen (PSMA) expression in prostate carcinoma with distant metastasis. Pathol Oncol Res. 2009;15:167-172.

9. Violet J, Jackson P, Ferdinandus J, et al. Dosimetry of ${ }^{177}$ Lu-PSMA-617 in metastatic castration-resistant prostate cancer: correlations between pretherapeutic imaging and whole-body tumor dosimetry with treatment outcomes. $J$ Nucl Med. 2019;60:517-523.

10. Current K, Meyer C, Magyar CE, et al. Investigating PSMA-targeted radioligand therapy efficacy as a function of cellular PSMA levels and intratumoral PSMA heterogeneity. Clin Cancer Res. 2020;26:2946-2955.

11. Gafita A, Calais J, Grogan TR, et al. Nomograms to predict outcomes after ${ }^{177} \mathrm{Lu}-\mathrm{P}$ SMA therapy in men with metastatic castration-resistant prostate cancer: an international, multicentre, retrospective study. Lancet Oncol. 2021;22:1115-1125.

12. Ferdinandus J, Violet J, Sandhu S, et al. Prognostic biomarkers in men with metastatic castration-resistant prostate cancer receiving [ ${ }^{177}$ Lu]-PSMA-617. Eur J Nucl Med Mol Imaging. 2020;47:2322-2327.

13. Vlachostergios PJ, Niaz MJ, Skafida M, et al. Imaging expression of prostatespecific membrane antigen and response to PSMA-targeted $\beta$-emitting 
radionuclide therapies in metastatic castration-resistant prostate cancer. Prostate. 2021;81:279-285.

14. Hofman MS, Emmett L, Sandhu S, et al. $\left[{ }^{177} \mathrm{Lu}\right]$ Lu-PSMA-617 versus cabazitaxel in patients with metastatic castration-resistant prostate cancer (TheraP): a randomised, open-label, phase 2 trial. Lancet. 2021;397:797-804.

15. Sandach P, Kersting D, Weber M, et al. PSMA- and FDG-PET mismatch assessment for optimized selection of PSMA radioligand therapy candidates [abstract]. Nuklearmedizin. 2021;60(suppl):P48.
16. Michalski K, Ruf J, Goetz C, et al. Prognostic implications of dual tracer PET/CT: PSMA ligand and $\left[{ }^{18} \mathrm{~F}\right] \mathrm{FDG}$ PET/CT in patients undergoing $\left[{ }^{177}\right.$ Lu]PSMA radioligand therapy. Eur J Nucl Med Mol Imaging. 2021;48: 2024-2030.

17. Czernin J, Sonni I, Razmaria A, Calais J. The future of nuclear medicine as an independent specialty. J Nucl Med. 2019;60(suppl):3S-12S.

18. Srinivas S, Iagaru A. To scan or not to scan: an unnecessary dilemma for PSMA radioligand therapy. $J$ Nucl Med. August 26, 2021 [Epub ahead of print]. 